\title{
Um Problema de Contorno Não-linear em Águas Subterrâneas ${ }^{1}$
}

J.A.N. BATISTA2, Departamento de Recursos Hídricos da FEC-UNICAMP, Av. Albert Einstein, 951, 13083-852 Campinas, SP, Brasil.

E. WENDLAND ${ }^{3}$, Departamento de Hidráulica e Saneamento, EESC-USP, Av. Trabalhador Sancarlense, 400, 13566-590 São Carlos, SP, Brasil.

\begin{abstract}
Resumo. A proteção contra poluição e a conservação dos mananciais de águas subterrâneas em níveis de cargas hidráulicas elevados por grandes territórios tem sido procurada no gerenciamento de recursos hídricos. Por isso, formulações aproximadas de novas condições de contorno não-lineares têm sido desenvolvidas na tentativa de estender a modelagem analítica na direção da grande variedade de problemas de escoamento matematicamente descritíveis em águas subterrâneas. Este trabalho equaciona o escoamento horizontal através da fronteira entre a área de afloramento e a área confinada de um aquífero, obtendo uma condição de contorno não-linear chamada de condição de Poincaré. A descontinuidade do potencial de descarga encontrada através da fronteira é especificada pela descontinuidade da função linha dublê. A aproximação do escoamento no contorno gera um sistema de equações singulares que exigiu um algoritmo híbrido que combina o método de NewtonRaphson e o da bisseção. A convergência do processo é sensível à estimativa inicial da solução, porém, em todos os casos estudados, linearizando-se a condição de contorno, produziu-se estimativas iniciais que sempre permitiram a convergência da solução. A solução obtida foi validada mediante a avaliação dos erros apenas sobre o contorno.
\end{abstract}

Palavras-chave. Escoamento em aquíferos; inomogeneidades; elementos analíticos; condição de Poincaré

\section{Introdução}

No campo dos modelos matemáticos de escoamento subterrâneo, a modelagem analítica tem-se desenvolvido com o objetivo de tornar possível a modelagem de áreas abertas e com variações contínuas de propriedades [6]. Essa abordagem está crescendo a cada novo problema de valor de contorno caracterizado em Águas Subterrâneas. A condição de contorno de uma área de afloramento expressa uma mudança forçada de escoamento de confinado para livre e vice-versa. Alguns trabalhos dedicados à formulação de problemas de campos potenciais sujeitos a condições de

\footnotetext{
${ }^{1}$ os autores agradecem à FAPESP pela bolsa (proc. n. 06/57242-2).

${ }^{2}$ nbatista@fec.unicamp.br

3 ew@sc.usp.br
} 
contorno não-lineares desenvolveram soluções exatas para o problema do escoamento potencial de um fluido ao redor de um cilindro poroso (e.g. [8]). Problemas não-lineares em aquíferos são, ainda, pouco estudados. Fitts e Strack [1] encontram a solução exata da passagem de um escoamento unidirecional e unidimensional sobre um degrau caracterizado sobre o embasamento do aquífero. Neste trabalho, é abordado de maneira aproximada um escoamento através de área de afloramento pela sobreposição de funções chamadas de linhas dublês.

\section{O Problema Físico}

A lei de Darcy descreve o vetor de vazão horizontal (descarga de volume de água no tempo por unidade de comprimento transversal à direção do escoamento) $\mathbf{Q}$ $\left[\mathrm{L}^{2} \mathrm{~T}^{-1}\right]$ para um dado potencial de descarga $\Phi\left[\mathrm{L}^{3} \mathrm{~T}^{-1}\right]$ na posição $\mathbf{r}[\mathrm{L}]$ como:

$$
\mathbf{Q}=-\frac{\partial \Phi}{\partial \mathbf{r}}
$$

O potencial expresso pela equação (2.1) descreve, de forma abrangente e indiscriminada, escoamentos livres e confinados. A equação (2.1) é igualmente contínua e diferenciável em todo o aquífero e permite, ainda, que desconfinamentos locais (Figura 1a), como ocorre sob lentes de argila, e confinamentos locais quando encobrem lentes de argila (Figura 1b) sejam tratados sem a necessidade da imposição de condições de contorno. A substituição da equação (2.1) na equação da continuidade da massa,

$$
\operatorname{div} \mathbf{Q}=0,
$$

torna o escoamento determinado completamente em termos de $\Phi$, o potencial de descarga em qualquer posição $\mathbf{r}$ pela seguinte equação governante:

$$
\nabla^{2} \Phi=0
$$

Segundo [4], o potencial para representar ambas as situações é dado pela seguinte expressão:

$$
\Phi=K(\phi-b)(H-b)-\frac{1}{2} K(H-b),
$$

sendo $K\left[\mathrm{LT}^{-1}\right]$ a condutividade hidráulica do aquífero, $\phi=p / \gamma+z[\mathrm{~L}]$ a carga hidráulica do escoamento (com a fração $p$, pressão manométrica, sobre $\gamma$, peso específico do fluido, sendo a carga de pressão e $z$ a carga de posição vertical); $H$ [L] é a altura do topo da camada aquífera e $b[\mathrm{~L}]$ é a altura da base.

Áreas de afloramento impõem que na fronteira entre as partes encoberta e descoberta haja mudança de escoamento, seja de livre para confinado, seja de confinado para livre (Figura 2).

O escoamento através da fronteira entre a porção livre $(l)$ e a porção confinada (c) deve satisfazer ao equilíbrio de descargas normais à fronteira, $\mathbf{Q}_{n}$ (utilizandose a equação (2.1) para a direção $\mathbf{n}$ normal à fronteira) e ao equilíbrio de cargas hidráulicas, $\phi$ (utilizando-se a equação (2.4) resolvida para $\phi$ ) na fronteira entre 


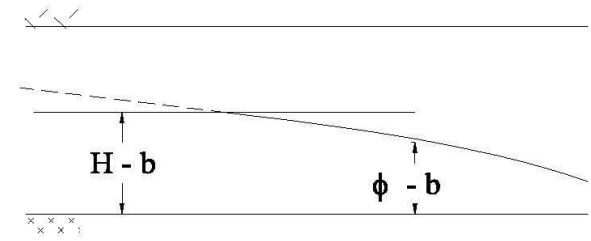

(a)

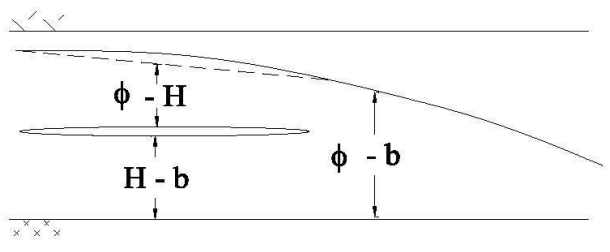

(b)

Figura 1: Situações de mudanças de escoamento não-forçadas. a) Desconfinamento sob lentes impermeáveis. b) Passagem do escoamento sobre uma lente impermeável.

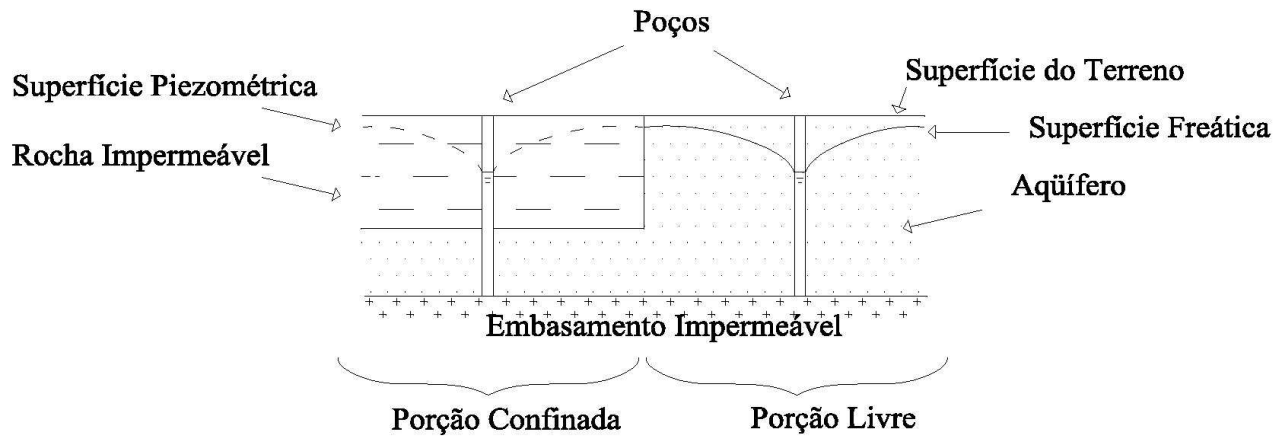

Figura 2: Perfil esquemático da fronteira entre uma região confinada e livre de um aquífero.

a porção livre e a confinada. A definição de potencial de descarga (equação (2.5)) fornece a igualdade de cargas e de descargas na fronteira com as seguintes expressões:

$$
\begin{aligned}
\frac{\partial \stackrel{c}{\Phi}}{\partial \mathbf{n}} & =\frac{\partial \stackrel{l}{\Phi}}{\partial \mathbf{n}} \\
\left(\frac{\stackrel{c}{\Phi}+\frac{1}{2} K H^{2}}{K H}\right) & =\frac{2 \stackrel{l}{\Phi}}{K} .
\end{aligned}
$$

Logo, a equação de Laplace (equação (2.3)) e as condições de contorno da equação (2.5) são que caracterizam o problema do escoamento em áreas de afloramento. O expoente quadrático das condições de contorno encontradas caracterizam este problema como um problema de Poincaré [8]. 


\section{Método de Solução}

A solução do problema do escoamento com fronteiras entre áreas livres e confinadas é obtida definindo-se uma variável chamada de pulo no potencial. A equação (2.4) demonstra que um mesmo valor de carga hidráulica em um ponto qualquer da fronteira de uma inomogeneidade se analizado imediatamente dentro $(+)$ e imediatamente fora da fronteira $(-)$, sendo $\phi^{+}=\phi^{-}$, produz diferentes potenciais, cuja diferença é chamada pulo no potencial, $\Phi^{+}-\Phi^{-} \neq 0$. Strack e Haitjema [7] formularam o uso de uma função linha dublê (função dublê integrada em um segmento de reta) para reproduzir o pulo de potencial necessário para equilibrar as cargas através de uma inomogeneidade. Seja o seguinte potencial complexo dado pelo dublê

$$
\Omega_{d}=\frac{1}{2 \pi} \frac{s}{z-z_{0}}
$$

que é uma função que atende a equação de Laplace, sendo $s$ o número complexo que fornece a intensidade e o sentido do dublê e $\Omega$ o potencial complexo cuja parte real vale $\Phi$ e a parte imaginária vale $\Psi$. Logo, pelo teorema de Cauchy-Riemann e pela lei de Darcy, diz-se que se $\Phi$ é o potencial de descarga, então $\Psi$ é a função de corrente do escoamento. Uma linha dublê é expressa pela integral de Cauchy sobre a função dublê como:

$$
\Omega_{l d}=-\frac{1}{2 \pi i} \int_{-1}^{1} \frac{\lambda(\Delta)}{Z-\Delta} d \Delta,
$$

sendo que $Z$ e $\Delta$ são, respectivamente, as coordenadas planas horizontais complexas locais sobre o domínio e sobre o segmento tais que as extremidades da linha dublê recaiam sobre o eixo dos reais entre -1 e $+1 ; \lambda$ é a função que distribui o esforço do dublê ao longo do segmento na direção ortogonal à do segmento, dada pelo complexo $-\frac{1}{i}$. Strack [5] e Jankovic e Barnes [2] apresentam representações polinomiais do esforço $\lambda$ em $Z=\Delta$, respectivamente na forma lagrangeana e na forma chebysheviana; e a solução da integral da equação (3.2). A aplicação da função dublê (equação (3.2)) no contorno é feita em segmentos de linhas retas definidas pelas coordenadas locais $Z$ e $\Delta$.

A expressão que permite resolver o escoamento no contorno é obtida da seguinte forma. Seja $T$ a transmissividade do aquífero, tal que:

$$
T=K h,
$$

em que $h$ assume o valor de $h=\phi-b$ no caso do escoamento estar livre e $h=H-b$ no caso do escoamento estar confinado. Utilizando-se a equação (3.3) e as definições de região dentro da fronteira $(+)$ e região fora da fronteira $(-)$, generaliza-se a a equação (2.5) ao redor da fronteira de forma a representar ambas as situações de escoamento, livre ou confinado, obtendo-se:

$$
\frac{\Phi^{+}+\frac{1}{2} K^{+}\left(H^{+}-b^{+}\right)^{2}}{K^{+}\left(H^{+}-b^{+}\right)}+b^{+}=\frac{\Phi^{-}+\frac{1}{2} K^{-}\left(H^{-}-b^{-}\right)^{2}}{K^{-}\left(H^{-}-b^{-}\right)}+b^{-} .
$$

Utilizando-se a definição de transmissividade (3.3), esta equação torna-se:

$$
\frac{\Phi^{+}+\frac{1}{2} T^{+}\left(H^{+}-b^{+}\right)}{T^{+}}+b^{+}=\frac{\Phi^{-}+\frac{1}{2} T^{-}\left(H^{-}-b^{-}\right)}{T^{-}}+b^{-} .
$$


Após isolar o pulo no potencial, $\Phi^{+}-\Phi^{-}$, e atribuir-lhe o esforço $\lambda$, tem-se:

$$
\lambda=\frac{T^{-}-T^{-}}{T^{-}} \Phi^{-}-\frac{T^{+}}{2}\left(H^{-}+b^{-}-H^{+}-b^{+}\right),
$$

em que a variável $H$ define a elevação do topo do aquífero com a possibilidade de $H=\phi$ se o escoamento tornar-se livre. Depois da expressão 3.6 ser avaliada em tantos pontos da fronteira (M) quantos forem os coeficientes de todos os elementos da fronteira $(\mathrm{N})$ produz-se um sistema de equações $\mathrm{MxN}$, com $\mathrm{M}=\mathrm{N}$. A situação de escoamento livre $(H=\phi)$ torna o sistema de equações 3.6 não-linear, pois pela definição de transmissividade (equação (3.3)) e pela definição de potencial em zonas livre (equação (2.4)) tem-se:

$$
T=\sqrt{2 K \Phi} .
$$

Isso torna a equação (3.6) um sistema de equações não-lineares singular, pois a equação (3.7) não pode ser avaliada em estimativas de solução em que $\Phi<0$. Métodos numéricos para resolver sistemas não-lineares singulares sofrem com regiões de gradientes muito grandes, ou mal definidos. Os métodos newtonianos, como métodos que utilizam os gradientes do sistema, formulam a solução do sistema por expressões da seguinte forma:

$$
\mathbf{a}_{k}=\mathbf{a}_{k-1}+\mathbf{W} \cdot \mathbf{f}\left(\mathbf{x}_{k-1}\right),
$$

sendo $\mathbf{a}_{k}$ a estimativa do vetor solução no passo $k, \mathbf{f}$ o vetor da função cuja raiz é desejada ( $\mathbf{f}=\mathbf{A a} \mathbf{a} \mathbf{b}$, com $\mathbf{A}$ para a matriz de coeficientes da variável $\mathbf{a}$ e $\mathbf{b}$ para o vetor independente da função) e $\mathbf{W}$ o controle de passo do processo iterativo baseado no gradiente da função $\mathbf{f}$. No caso do método de Newton-Raphson, $\mathbf{W}$ vale menos o inverso da matriz jacobiana, $\mathbf{W}=-\mathbf{J}^{-1}$. Por outro lado, o método da bisseção é amplamente aceito como método de estimativa da solução e possui convergência incondicional, mas lenta. Alternativamente, em problemas não-lineares da área de Engenharia, a rápida convergência dos métodos de gradientes tem sido associada com frequência à possibilidade de escapar de singularidades do método da bisseção (e.g. [3]). Numa tentativa de substituir as estimativas singulares, foi elaborado um algoritmo que utiliza o método de Newton-Raphson mas recorre ao método de bisseção sempre que 1$)$ a estimativa $\left(\mathbf{a}_{\mathbf{k}}\right)$ levar algum elemento da função $(\mathbf{f})_{i}$, para qualquer $i \leq M$, a atravessar o eixo zero, seja do positivo para o negativo, seja o contrário; ou 2) a tentativa $\left(\mathbf{x}_{\mathbf{k}}\right)$ leva $\Phi$ ao valor de $\Phi<0$. O algoritmo foi concebido da seguinte maneira:

1. Adote a solução inicial $\left(\mathbf{a}_{k=0}\right)$.

2. Estime as raízes $\left(\mathbf{a}_{k=1}\right)$ da função (f) pelo método de Newton-Raphson.

3. Se a k-ésima estimativa de $\mathbf{a}\left(\mathbf{a}=\mathbf{a}_{k}\right)$ faz alguma componente de $\mathbf{f}$ mudar de sinal e, portanto, $\left[\mathbf{f}\left(\mathbf{a}_{k}\right)\right]_{i} *\left[\mathbf{f}\left(\mathbf{a}_{k-1}\right)\right]_{i}<0$ para qualquer $i<M$ ou a função $\mathbf{f}$ for singular em $\mathbf{a}_{\mathbf{k}}$, então estime as raízes entre $\mathbf{a}_{\mathbf{k}}$ e $\mathbf{a}_{\mathbf{k}-\mathbf{1}}$ tomando-se $\mathbf{a}_{\mathbf{k}+\mathbf{1}}$ $=0,5 * \mathbf{a}_{\mathbf{k}-\mathbf{1}}+0,5 * \mathbf{a}_{\mathbf{k}}$.

4. Repita o passo 3) até que $\mathbf{a}_{\mathbf{k}}$ e $\mathbf{a}_{\mathbf{k - 1}}$ produzam $\left[\mathbf{f}\left(\mathbf{a}_{k}\right)\right]_{i} *\left[\mathbf{f}\left(\mathbf{a}_{k-1}\right)\right]_{i}>0$ para todo $i<M$ e que a função $\mathbf{f}$ seja avaliada somente com componentes reais e finitas. 
5. Se $\|\mathbf{f}\|$ for maior que o valor tolerado e a quantidade de estimativas $(k)$ for menor que a tolerada, prossiga a partir do passo 2 , caso contrário pare.

Esse algoritmo parte da solução inicial para obter estimativas pelo método de Newton. Mediante a mudança de sinal ou a presença de singularidade nas componentes da função, um falso método de bisseção $\left(\mathbf{a}_{\mathbf{k}-1}=0,5 * \mathbf{a}_{\mathbf{k - 1}}+0,5 * \mathbf{a}_{\mathbf{k}}\right)$ é acionado no item 3 do algoritmo. O método recebe esse nome devido à semelhança com o mecanismo do método da bisseção e à utilidade diferenciada do método para distanciar a avaliação da raiz ao invés de aproximá-la, como estabelece o método da bisseção canônico. Trabalhos recentes voltados para aumento da taxa de convergência de processos iterativos para solução de sistemas não-lineares afirmam que a combinação de métodos newtonianos ao método de bisseção produz resíduos de estimativas, $\left(\mathbf{a}_{\mathbf{k}}\right)_{i}-\left(\mathbf{a}_{\mathbf{k - 1}}\right)_{i}$ para qualquer $i<M$, a taxas quadráticas [9] e cúbicas [3], dependendo do comportamento do controle de passos. Em ambos os trabalhos, [3] e [9], foram estudadas funções de uma variável apenas.

\section{Resultados e Discussões}

Foi elaborado um problema hipotético em que um campo de escoamento unidirecional produzido a uma distância infinita atravessa uma inomogeneidade definida em uma área finita. As unidades de comprimento e de tempo são fictícias e são: "u"para a unidade de comprimento [L] e "t"para a unidade de tempo [T]. Portanto, as unidades de carga hidráulica, $\phi$, cota de elevação do topo, $H$ e da base, $b$, são dadas por "u". A condutividade hidráulica, $K$, é dada por "u/t". A inomogeneidade apresenta uma redução na cota de elevação do topo do aquífero (Figura 3). O escoamento ocorre livre fora da inomogeneidade (assinalada com sobrescritos " -") e passa a confinado dentro da inomogeneidade (assinalada com sobrescritos "+").

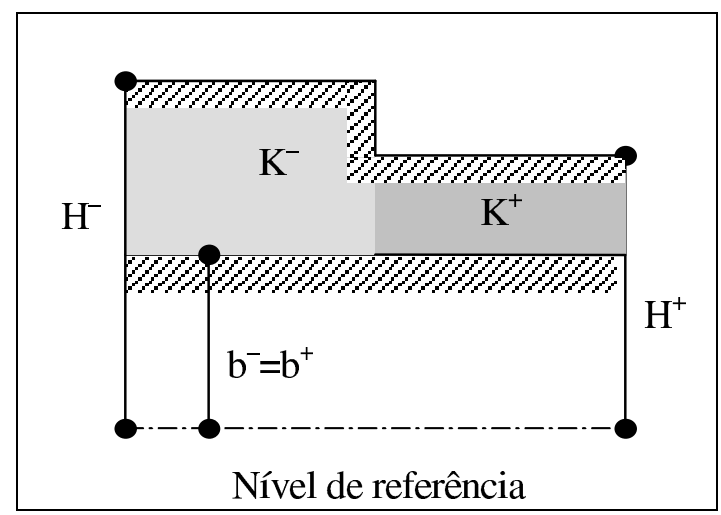

Figura 3: Corte esquemático da inomogeneidade do primeiro problema.

Foram estudadas três configurações de elevação do topo para esse primeiro problema, conforme resumidas na Tabela 1. Em todas as configurações as condutividades hidráulicas são iguais. 
Tabela 1: Parâmetros hidrogeológicos do aquífero para o primeiro problema.

\begin{tabular}{|c|c|c|c|}
\hline \multicolumn{2}{|c|}{ Configuração 1 } & \multicolumn{2}{c|}{ Configuração 2} \\
\hline \hline$K^{-}=1,0 u / t$ & $K^{+}=K^{-}$ & $K^{-}=1,0 u / t$ & $K^{+}=K^{-}$ \\
\hline$b^{-}=-1,0 u$ & $b^{+}=-1,0 u$ & $b^{-}=-1,0 u$ & $b^{+}=-1,0 u$ \\
\hline$H^{-}=9,5 u$ & $H^{+}=8,5 u$ & $H^{-}=9,5 u$ & $H^{+}=1,1 u$ \\
\cline { 2 - 2 } & \multicolumn{2}{|c}{ Configuração 3} \\
\cline { 2 - 3 } \\
\cline { 2 - 3 } & $K^{-}=1,0 u / t$ & $K^{+}=K^{-}$ \\
\cline { 2 - 3 } & $b^{-}=-1,0 u$ & $b^{+}=-1,0 u$ \\
\cline { 2 - 3 } & $H^{-}=9,5 u$ & $H^{+}=0,1 u$ \\
\hline
\end{tabular}

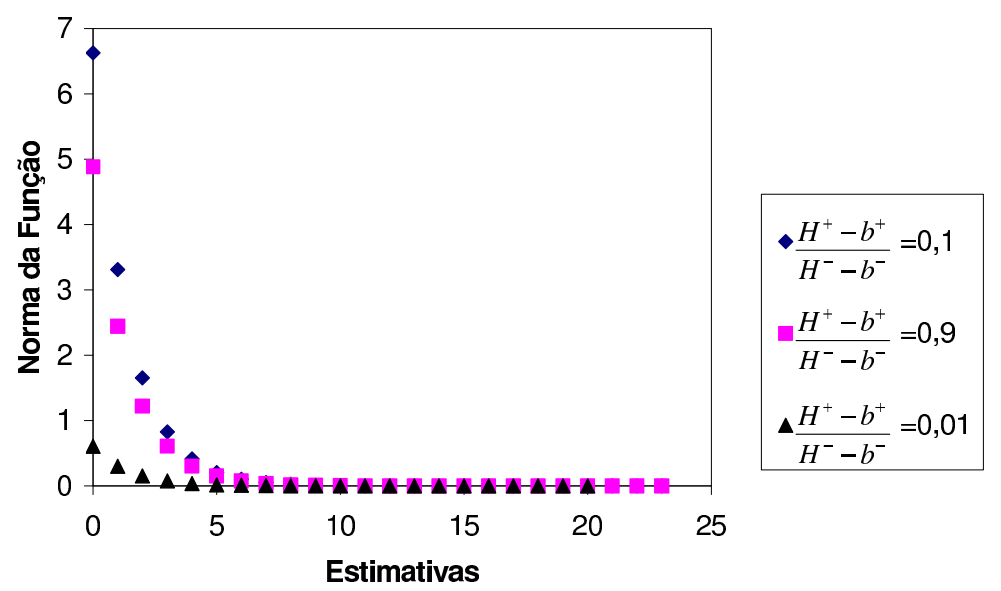

Figura 4: Evolução da solução do primeiro problema.

Pela definição da equação (3.3), uma diferença de transmissividade devido a $\phi^{+}-b^{+} \neq \phi^{-}-b^{-}$pode ser convertida em uma outra devido a $K^{\prime+} \neq K^{\prime-}$. Assim, quando o escoamento é confinado, $\phi \geq H$, tem-se:

$$
\frac{K^{\prime+}}{K^{\prime-}}=\frac{H^{+}-b^{+}}{H^{-}-b^{-}} \text {. }
$$

A solução inicial de cada configuração é obtida da linearização da transmissividade assumindo $K^{\prime+}=K^{+}$e $K^{\prime-}=K^{\prime+} \frac{H^{+}-b^{+}}{H^{-}-b^{-}}$. Cada simulação foi obtida por quatro linhas dublês (equação (3.2)) que geraram um sistema matricial não-linear do tipo da equação (3.6) com sete equações e sete incógnitas. Em cada configuração da cota de elevação do topo a evolução do processo converge para a solução conforme ilustrado na Figura 4.

As estimativas iniciais produziram diferentes normas iniciais $\left\|\mathbf{f}_{\mathbf{0}}\right\|$. A Configuração 2 foi a que produziu a maior norma inicial. Embora a Configuração 1 tenha sido a de menor diferença de transmissividades, não foi a de menor norma inicial. 
Por outro lado, a maior diferença de transmissividades (Configuração 3) produziu a menor norma inicial. Isso aconteceu porque a redução de transmissividade na inomogeneidade reduz a vazão através da fronteira, que se reflete em um aumento da carga hidráulica no domínio. Com isso, as condutividades hidráulicas $\left(K^{+}\right.$e $\left.K^{-}\right)$ calculadas para a condição semelhante da Configuração 3 guardaram uma melhor equivalência com as transmissividades das espessuras resultantes da simulação (se $H^{+} \leq \phi^{+}$então $h^{+}=H^{+}-b^{+}$, caso contrário $h^{+}=\phi^{+}-b^{+}$; se $H^{-} \leq \phi^{-}$então $h^{-}=$ $H^{-}-b^{-}$, caso contrário $\left.h^{-}=\phi^{-}-b^{-}\right)$. As cargas hidráulicas $(\phi)$ se posicionaram em cotas mais próximas do topo do aquífero $\left(H^{+}\right)$e da inomogeneidade $\left(H^{-}\right)$. Os experimentos demonstraram que mesmo o processo iniciando a diferentes distâncias da solução e percorrendo quantidades de passos diferentes, a ordem de convergência permaneceu a mesma. Nas três simulações a ordem de convergência $m$ do processo de solução, tal que $\left\|\mathbf{f}_{\mathbf{n}}\right\|=\kappa\left\|\mathbf{f}_{\mathbf{n}-\mathbf{1}}\right\|^{m}$, sendo $\left\|\mathbf{f}_{\mathbf{n}}\right\|$ a norma da função vetorial $\mathbf{f}$ no passo $n$, foi aproximadamente linear com a constante de proporcionalidade $\kappa=0,5$.

\section{Conclusões}

As condições de contorno do escoamento em áreas de afloramento demonstram a dependência não-linear das cargas piezométricas do escoamento dentro e fora de inomogeneidades. Matematicamente, o escoamento por inomogeneidades que forçam o escoamento a mudar entre livre e confinado recai em um problema de valor de contorno não-linear expresso pela condição de Poincaré para a equação diferencial linear de Laplace. Assim, este trabalho descreve o escoamento horizontal em áreas de afloramento por intermédio da equação de Laplace e de uma condição de contorno não-linear classificada como condição de Poincaré. A implementação da solução deste problema exigiu a elaboração de um algoritmo iterativo híbrido que combina o método de Newton-Raphson ao da bisseção. Análises de sensibilidade foram obtidas a partir de um problema hipotético. Foram resolvidas três situações de mudança de escoamento forçada a partir de condições iniciais obtidas por intermédio de uma equivalência hidráulica aparente na transmissividade. O comportamento do processo iterativo demonstrou-se convergente a uma razão constante e igual a $\frac{1}{2}$ e sempre estável. A validação da solução é garantida porque a função linha dublê satisfaz a equação governante (equação de Laplace) e, também, pelo ajuste obtido para as condições de contorno. A aplicação das condições de Poincaré em problemas práticos em trabalhos futuros permitirá avaliar questões transfronteiriças como a interferência bilateral do bombeamento de áreas de afloramento e de áreas confinadas.

\footnotetext{
Abstract. Issues in groundwater management include to protect aquifers against pollutants and to keep piezometric heads at high levels in aquifer wide. Therefore, studies in groundwater mechanics is still interested in boundary value problems, since the flow problems have enlarged study areas and found new non-linear frontiers. Approximate solutions are such a convenient approach since they allow the mathematical description of phenomena. This work formulates the horizontal groundwater flow through boundaries between unconfined and confined areas of aquifers, finding a Poincaré boundary condition. The jump in discharge potential met at the boundary is specified with the line-doublet function discontinuity. The
} 
boundary approximation generated a singular system of equations whose solution was possible hybridizing the Newton-Raphson method with the bisection method. The iterative process was convergent to all the tests if the initial estimate is obtained by means of a simple linearization of the boundary condition. The solution was validated by means of the error at the boundary.

\section{Referências}

[1] C.R. Fitts, O.D.L. Strack, Analytic solutions for unconfined groundwater flow over a stepped base, Journal of Hydrology, 177 (1996), 65-76.

[2] I. Jankovic, R. Barnes, High-order line elements in modeling two-dimensional groundwater flow, Journal of Hydrology, 226 (1999), 211-223.

[3] P.K. Parida, P.K. Gupta, A cubic convergent iterative method for enclosing simple roots of nonlinear equations, Applied Mathematics and Computation, 187 (2007), 1544-1551.

[4] O.D.L. Strack, Flow in aquifers with clay lamina, 1. The comprehensive potential, Water Resources Research, 17 (1981), 985-992.

[5] O.D.L. Strack, "Groundwater Mechanics", Prentice-Hall, Englewood Cliffs, 1989.

[6] O.D.L. Strack, The development of new analytic elements for transient flow and multiaquifer Flow, Ground Water, 44 (2006), 91-98.

[7] O.D.L. Strack, H.M. Haitjema, Modeling double aquifer flow using a comprehensive potential and distributed singularities 2. Solution for inhomogeneous permabilities, Water Resources Research, 17, (1981), 1551-1560.

[8] L. Von Wolfersdorf, W. Mönch, Potential flow past a porous circular cylinder, Zeitschrift fur Angewandte Math. und Mech., 80, (2000), 457-471.

[9] X. Wu, D. Fu, New high order convergence iteration methods without employing derivatives for solving nonlinear equations, Computers 8 Mathematics with Applications, 41, (2001), 489-495. 